\title{
Deuterium Inventory in Tore Supra: status of post mortem analyses
}

\author{
T. Dittmar ${ }^{\text {a, }}$, P. Roubin ${ }^{\mathrm{d}}$, E. Tsitrone ${ }^{\mathrm{a}}$, E. Gauthier ${ }^{\mathrm{a}}$, A. Hakola ${ }^{\mathrm{c}}$, J. Likonen ${ }^{\mathrm{c}}$, F. Linez $^{\mathrm{a}}$, C. \\ Martin $^{d}$, M. Mayer ${ }^{b}$, C. Pardanaud ${ }^{d}$, JY Pascal ${ }^{a}$, B. Pasquet ${ }^{e}$, B. Pégourié ${ }^{a}$, J. Roth ${ }^{b}$, I. \\ Roure $^{e}$, R. Ruffe ${ }^{d}$ \\ ${ }^{a}$ Association Euratom-CEA, CEA/DSM/IRFM, CEA Cadarache, 13108 Saint-Paul-lès-Durance, France \\ ${ }^{b}$ MPI für Plasmaphysik, EURATOM Association, Boltzmannstr. 2, 85748 Garching, Germany \\ ${ }^{c}$ Association EURATOM-TEKES, VTT, P O Box 1000, 02044 VTT, Espoo Finland \\ ${ }^{d}$ PIIM,UMR 6633 CNRS/Université de Provence, 13397 Marseille, France \\ ${ }^{e}$ CEA-DEN-CAD-DEC, CEA Cadarache, F-13108 Saint Paul-lez-Durance, France
}

\section{PACS numbers:}

\author{
52.40.Hf Plasma-material interactions; \\ boundary layer effects
}

\subsection{Fa Tokamaks, spherical tokamaks}

${ }^{*}$ Corresponding author. E-mail address: Timo.Dittmar@cea.fr

\begin{abstract}
A dedicated study on fuel retention has been launched in Tore Supra which included a $D$ wallloading campaign and the dismantling of the main limiter (Deuterium Inventory in Tore Supra, DITS project). This paper presents new results of post mortem analyses performed on selected limiter tiles. Scanning electron microscopy shows thick layers with poloidally oriented tip-shaped structures in deposition zones. In the erosion zone deposits inside the open porosities of the bulk material of the tiles were found. Raman microscopy indicates that hard deuterated amorphous carbon layers are deposited on the limiter tiles and on their gap sides. Secondary ion mass spectrometry $D$ depth profiles show an increase of the $D$ content near the bottom of the gap, in agreement with previous results. The campaign markers $\left({ }^{13} \mathrm{C}\right.$ and $\left.{ }^{11} \mathrm{~B}\right)$ are found in only 2 out of 7 measurements in the deposited layers. Finally, the agreement of the SIMS data with previous NRA measurements is discussed, especially discrepancies possibly due to the non-uniformity of the samples.
\end{abstract}

\section{Introduction}

The use of carbon as plasma facing material allows efficient heat extraction while maintaining acceptable plasma contamination due to its good thermo-mechanical properties and low atomic mass. Nevertheless, carbon can lead to significant in-vessel fuel retention in fusion devices: the extrapolation of present results shows that the safety limited inventory of releasable tritium in ITER $(700 \mathrm{~g})$ could be reached within 100-1000 nominal discharges (400 s, full power 50:50 DT plasmas) [1]. With its ability to perform discharges of relevant duration with actively cooled plasma facing components (PFCs), Tore Supra offers a unique opportunity to address this issue in true steady state from the plasma-wall interaction point of view.

Recent progress was achieved in this field with the DITS project, aimed at better understanding fuel retention in tokamaks [2, 3]. The project includes 3 phases: a dedicated campaign to load the vessel walls with deuterium, the dismantling of a sector of the Toroidal Pump Limiter (TPL) to extract selected samples, and an extensive sample analysis program in collaboration with European partners in the frame of the EU Plasma-Wall Interaction Task 
Force. For the $\mathrm{D}$ wall-loading experiments, more than 160 long (> $1 \mathrm{~min}$ ), identical discharges were performed for a total time of $\sim 5 \mathrm{~h}$ of plasma. $\mathrm{A}{ }^{13} \mathrm{C}$ carbonisation and $\mathrm{a}{ }^{11} \mathrm{~B}$ boronisation were performed by glow discharge prior to the $D$ wallloading program, to mark the start of the campaign for post mortem analyses [2]. From gas balance measurements, a constant D-retention rate was measured $\left(\sim 1.7 \times 10^{20} \mathrm{D} \mathrm{s}^{-1}\right.$, taking into account recovery between shots, long term outgassing, etc.), with no sign of wall saturation, leading to a total increment of the wall inventory of $\sim 3.1 \times 10^{24} \mathrm{D}$ [2].

First measurements of the D-content were performed on a selected set of 10 tiles extracted from the TPL, using combined surface analysis methods [2] [3]: local measurements with Nuclear Reaction Analysis (NRA) and integrated measurements with Thermo-Desorption Spectrometry (TDS), to estimate the relative contribution of the different zones of interest on the TPL (erosion, thick deposits, thin deposits, see Figure 1). They showed that the post mortem inventory is mainly due to co-deposition (90\% of the total), in particular due to deposits in the castellated structures between tiles. However, D was also detected deep in the material in the erosion zone (10\% of the total), which is attributed to bulk diffusion inside the porous network of the Carbon Fiber Composite (CFC) used for the TPL tiles [3]. In average, a surface D-concentration of $5 \%$ was measured in the eroded zone, and $\sim 20$ $\%$ in the deposited zone with a typical thickness of $15-20 \mu \mathrm{m}$. Extrapolated over the whole TPL, this corresponds to $\sim 50 \%$ of the DITS increase of the wall inventory deduced from gas balance $\left(1.5 \times 10^{24}\right.$ D).

In addition to TDS and NRA, Secondary lon Mass Spectrometry (SIMS) has been used for D and impurity contents. Scanning Electron Microscopy (SEM) and Raman microscopy have also been applied for structural analysis. The aim of this paper is to review these new analyses and to discuss the consistency among the various results, especially concerning the $\mathrm{D}$ content.

\section{Experimental background}

\subsection{The Tore Supra toroidal pump limiter}

After the DITS campaign, one sector of the TPL was extracted from Tore Supra. Figure 1 shows a picture of this sector and the complex erosion/deposition pattern resulting from plasma operation [2]. Erosion zones are found in the most heavily plasma loaded areas, while thin deposits are found in the area shadowed from the plasma due to the magnetic ripple structure, as well as in the plasma loaded area corresponding to the far scrape-off layer (SOL) on the low field side of the limiter. Thick deposits are found in the boundary between the plasma loaded and the plasma shadowed zones. The first set of tiles selected for analysis in the different zones is also shown in Figure 1. The finger and tile numbering scheme is also indicated (FxTy for tile $y$ on finger $x$ ).

\subsection{Post mortem analysis techniques}

To assess the $D$ inventory of the limiter, the TPL tiles were cut in quarters [3] and the resulting samples were analyzed with different $D$ sensitive techniques, including TDS, NRA and SIMS. The TDS and NRA set-ups are described in [2] [3]. In particular, NRA was performed using incident beam energies ranging from 800 $\mathrm{keV}$ to $6 \mathrm{MeV}$, probing the material down to a depth of $~ 35 \mu \mathrm{m}$ [9]. The D-content extrapolated from the local $D$ depth profiles measured with NRA were within $30 \%$ of the integrated $D$ content measured with TDS except for thick deposits where the thickness of the deposits exceeded the NRA depth penetration [3].

In addition, SIMS measurements [4] were performed to obtain, along with the $D$ depth profile, impurity depth profiles (metals, oxygen) as well as the DITS campaign marker depth ranges $\left({ }^{13} \mathrm{C}\right.$ and $\left.{ }^{11} \mathrm{~B}\right)$. The SIMS measurements were performed in two laboratories: at LECA in CEA Cadarache (France) using a CAMECA 6F and at the Technical Research Centre Finland (VTT) using a VG IX-70S. The good mass resolution at LECA allowed detailed studies of the ${ }^{13} \mathrm{C}$ marker distribution, while the high sputtering speed at VTT allowed the analysis of the thick deposited layers. To estimate the depth scale, the SIMS sputtering craters were characterized, using confocal microscopy for samples analyzed at LECA and profilometry for those analyzed at VTT (typical sputtering depths from 3 to $65 \mu \mathrm{m}$ ).

Samples from the three different zones (see tiles indicated in Figure 1) were also studied using SEM and Raman microscopy. SEM was performed at the Centre de Microscopie and at the CP2M (Universités d'Aix-Marseille, France) using a Philips XL 30 microscope. Raman microscopy was performed using a LabRAM HR Horiba Jobin-Yvon (excitation wavelength: $532 \mathrm{~nm}$, incident power: < $1 \mathrm{~mW}$, spatial resolution: $\left.\sim 1 \mu \mathrm{m}^{2}\right)$. The Raman probe depth is estimated between 40 and $100 \mathrm{~nm}$.

\section{Results and discussion}




\subsection{Scanning electron microscopy}

Figure 2 shows SEM images obtained from the plasma facing top of the thick deposit tile (Figure 2a-b), the poloidal gap side of the thick deposit tile (Figure 2c), the plasma facing top of the thin deposit tile (Figure 2d), and the plasma facing top of the eroded tile (Figure 2e-f). The orientation of the tile top images is consistent with the tile position in Figure 1 (e.g. the high (low) field side corresponding to the bottom (top) of the picture). Significant flaking is observed for the thick tile top deposit (Figure 2a-b).

As previously observed on neutraliser deposits [5], tip-shaped structures are observed on the top of the thick and thin deposit tiles, as well as on the gap sides, while they are not seen on the top of the eroded tile. The tips are locally parallel, lying on the tile surface. For all the surfaces observed here, the tips are oriented roughly in the poloidal direction and are pointing to the low field side. This "tipped morphology" and the effect of flaking deposits lead to rough surfaces. The tip sizes vary widely: tips are larger in zones with strong deposition, e.g. for the gap side of the thick deposit tile (Figure 2c), and smaller in zones with low deposition, e.g. for the thin deposit tile (Figure 2d). Along the gap surfaces, the mean tip size decreases regularly (Figure 2c) from the top towards the bottom with no tips being visible below $1 \mathrm{~mm}$. The size of the surface morphology features varies from a few $\mu \mathrm{m}$ (small tips) to a few hundreds $\mu \mathrm{m}$ (large tips, flakes). Large porosities are observed on the top of the thin deposit tile (Figure 2d), corresponding to the intrinsic porosity of CFC, for example at fiber bundle boundaries. The porosity and the CFC fiber bundle network are still clearly visible on the top of the eroded tile, with thick and layered deposits on each gap side. Figure $2 e$ shows the side of an eroded tile (F27T10) seen from above. Three different zones can be identified (1) vacuum, (2) deposits on the gap, and (3) the top of the tile itself. The interface between the layer deposited at the gap surface and the tile shows a long crack, indicating that the gap deposits are poorly attached. The deposit thickness at the top of the gap can be estimated to $\sim 400 \mu \mathrm{m}$ at the top of the two poloidal gaps, and $170 \mu \mathrm{m}$ and $\sim 60 \mu \mathrm{m}$ for the toroidal gaps, for the low and high field side, respectively. This toroidal asymmetry could be related to $E \times B$ drift, as seen in simulations of plasma deposition in gaps

\section{[6].}

Figure $2 f$ shows the bundles of fibers, parallel and perpendicular to the top surface of the tile, with the pyrolytic matrix around them. Figure $2 f$ also clearly shows deposition inside the open porosities existing in the virgin CFC (indicated by the arrows), these porosities being nevertheless not totally filled. This supports previous studies [7][8], showing that the $D$ trapping sites are linked to the presence of open porosities: the $D$ content deep inside the material in the erosion zone could then be due to co-deposition inside the CFC porous network [2].

\subsection{Raman microscopy}

Figure 3 shows examples of Raman spectra recorded from different locations $\left(1 \mu \mathrm{m}^{2}\right.$ spot) on the top and on the gaps of tiles from the three types of zones. The correlation between the carbon structure and the location of the deposit is not obvious and is still under study. Most of them were typical of hard layers (lower curve of

Figure 3), i.e. amorphous carbon. The spectra nevertheless strongly varied from spot to spot, indicating that the deposits are very inhomogeneous, some spectra being even close to those of disordered graphite-like carbon (upper curve of

Figure 3) with all the intermediate states possible (two middle curves). This can be due to the different temperatures the samples have been exposed to or due to different precursors reaching the samples. An estimation of their $D$ content using Raman microscopy has been proposed [10] by measuring the ratio $\eta$ between the slope of the photoluminescent background and the height of the main band ( $G$ band).

Figure $3 b$ shows $\eta$ as a function of the $G$ band Full Width at Half Maximum (FWHM) for various positions on the top of the thick deposit tile, a G band FWHM larger than $100 \mathrm{~cm}^{-1}$ corresponding to amorphous carbon [11]. Most of the $\eta$ values obtained here are between 1 and $5 \mu \mathrm{m}$ and would correspond to a D-content between 22 and 35 at. \%, not far from NRA estimates. The large scattering of the data indicates that the D-content is also very nonuniform. Note that this estimation of the $D$ content is preliminary and that temperature effects should be taken into account for quantitative results

[12].

\subsection{Comparison between NRA and SIMS}

The consistency between the global and local $D$ inventory measurements by TDS and NRA was reported in [3]. Therefore we will now focus on the comparison between the local SIMS and NRA profile measurements. 
The analysis of the NRA spectra provides the D content as a function of depth expressed by area density of atoms in the solid [13], while SIMS records the mass-spectra of species eroded by the probing beam as a function of sputtering time. In both cases, assumptions are needed to rescale the results as a function of depth. To transform area density of atoms into spatial depth, the geometric mass densities of CFC and the deposits are needed. Densities from $1.5 \mathrm{~g} / \mathrm{cm}^{3}$ for soft, hydrogen-rich films, up to $2.4 \mathrm{~g} / \mathrm{cm}^{3}$ for hard, carbon-rich films are reported for a-C:H films [14] (depending on $\mathrm{H} / \mathrm{C}$ ratio of the incoming flux, substrate temperature, energy of the incident ions, etc.). Consistently with the $D / C$ ratio measured by NRA and previous studies, a density of $1.8 \mathrm{~g} / \mathrm{cm}^{3}$ is assumed for deposits, as well as for the CFC material (manufacturer specification for NB11). The influence of this parameter on the NRA profiles is shown in Figure 4 with horizontal error bars corresponding to densities between 1.6 and 2.2 $\mathrm{g} / \mathrm{cm}^{3}$.

In the case of SIMS, crater depth measurements are used to estimate the beam sputtering rate and then to derive the depth profile. The main source of uncertainty is due to the surface roughness of the samples $(\sim 5 \mu \mathrm{m}$ for virgin CFC, up to $100 \mu \mathrm{m}$ for the flaking deposited layers). For the VTT set-up, sputtering rates between 0.5 and $1.3 \mathrm{~nm} / \mathrm{s}$ were measured on eroded and deposited samples, with a large scattering of the data for both CFC and deposits. Based on these measurements an average value of $1.2 \mathrm{~nm} / \mathrm{s}$ was used for calculating the VTT SIMS depth scale on samples where the crater depth estimation was not possible. For the LECA set-up, the crater depth was hardly measurable, in particular for samples with deposits. Therefore only the VTT data was used for the comparisons of the $\mathrm{D}$ depth distribution.

Finally, the SIMS data obtained are not quantitative, and the $\mathrm{D} / \mathrm{C}$ signal ratios shown in the following section are given in arbitrary units, using a fixed rescaling factor on all graphs for comparison with NRA data.

\subsubsection{SIMS and NRA data for top samples}

Figure 4 shows D/C profiles obtained from SIMS (VTT) and NRA on samples from the different zones of interest where data from both techniques were available (note that SIMS and NRA were performed on different quarters of the same tile). SIMS-1 and SIMS-2 correspond to different locations on the same sample. As can be seen from Figure 4, the agreement between SIMS and NRA varies significantly This might be due to the strong non-uniformity of the samples at the micrometer scale, as presented in the structural analysis in section 3.1, and to the different probing sizes of the methods used. Indeed, as illustrated in
Figure 2b, NRA measurements integrates over typically a $1 \times 1 \mathrm{~mm}^{2}$ spot, which is larger than the typical scale of the different sample features (deposited tips, porosities, flaking), while SIMS is much more local $\left(100 \times 130 \mu^{2}\right.$ actively analyzed area for the VTT SIMS set-up). This can also explain the different levels of agreement between SIMS and NRA for the different types of deposits: on the relatively homogenous thin deposits, the NRA data and SIMS measurements at different locations agree well whereas in the case of the thick deposits, SIMS measurements at different locations can probe very different deposits (Figure 4a). The same explanation could be used to explain the large difference in the SIMS/NRA D profiles in the erosion zone: The NRA measurements average over a larger area of the eroded samples and therefore the localized depositions in the open porosities (compare Figure $2 \mathrm{e}$ ) of the CFC material always contribute to the NRA D signal whereas SIMS with its much smaller probing area can sees inhomogeneous samples. Other effects which contribute to uncertainties in the depth scales and the relative signals strengths in the SIMS measurements, e.g. matrix effects or ion beam mixing, are considered to have less influence than the surface roughness and the intrinsic inhomogeneity of the deposits. However, as the number of measurements is still scarce (the number of samples analyzed with both VTT-SIMS and NRA is only seven so far), no definitive conclusions can be drawn at this stage.

\subsubsection{SIMS and NRA data for gap samples}

In addition to the top surface of the tiles, the $D$ distribution along the gap side surfaces has also been studied with both SIMS and NRA. NRA data showed that the D content decreased as a function of the distance from the plasma facing entrance of the gap, and increased again at the bottom of the gap [3]. As seen from Figure 5, showing the $\mathrm{D}$ distribution along a gap surface, this trend is confirmed here by the SIMS data. In both cases, each $D$ depth profile was integrated to derive the total $D$ content for the different locations along the gap, from the plasma exposed entrance to the bottom of the sample at $6 \mathrm{~mm}$. This behavior was observed both for "bottom less" poloidal and for the castellated toroidal gaps. Similar observations regarding the $D$ profiles along the gap surfaces were made in TEXTOR [15] and DIII-D ([16] increase visible in the figures for $D$ contribution, not explicitly mentioned in the text). Using the $D$ depth profiles obtained by NRA, the decrease in the $D$ content in the gap can be attributed to both a decrease in the $\mathrm{D} / \mathrm{C}$ ratio measured and 
a decrease in the deposited layer thickness. The layer thickness increases again near the bottom of the gap with a correspondingly increased D content.

\subsubsection{Search for the ${ }^{13} \mathrm{C}$ and the ${ }^{11} \mathrm{~B}$ markers of the DITS campaign}

${ }^{13} \mathrm{C}$ and ${ }^{11} \mathrm{~B}$ layers were deposited in Tore Supra to mark the beginning of the DITS campaign and to estimate the relative DITS and pre-DITS contributions, especially for the $D$ inventory. The LECA SIMS set-up offered a sufficient mass resolution to distinguish ${ }^{13} \mathrm{C} /{ }^{12} \mathrm{CH}$ and ${ }^{11} \mathrm{~B} /{ }^{10} \mathrm{BH}$ and was therefore used for this analysis. Five tiles $(2$ in the erosion, 1 in the thin deposit and 2 in the thick deposit zones) were analyzed, each at 2 to 4 different locations on the top surface of the tile. As expected, no remnants of the marker layers were found on the samples in the erosion zones. But most of the samples in the deposition zones showed no clear evidence of the ${ }^{13} \mathrm{C}$ and ${ }^{11} \mathrm{~B}$ markers either. Recognizable peaks in the ${ }^{13} \mathrm{C} /{ }^{11} \mathrm{~B}$ could only be found in two measurements: in 1 outof 3 measurements on F17-T7 (thin deposits) and 1 out of 2 on F26-T3 (thick deposits). Measurements on the 3 other tiles did not show pronounced peaking in the ${ }^{13} \mathrm{C} /{ }^{11} \mathrm{~B}$ signals. This could be again due to the strong non-uniformity of the samples at the SIMS scale or due to continuous re-erosion/re-deposition of the layers during plasma operation [17]. This continuous remixing of the material could spread the markers and dilute them below the detection limits. Further investigation of the DITS start markers is needed in the next post-mortem analysis campaign.

\section{Summary}

The first post mortem analysis campaign of the DITS project allowed gaining insight on fuel retention in Tore Supra [3] and new results from structural analyses and SIMS measurements are now available. The structural analysis shows that the deposited layers exhibit a tip-shaped structure, which is preferentially oriented. Thick deposits (a few 100's $\mu \mathrm{m}$ ) have been observed in the gaps. In the erosion zone, deposits are seen in the open porosities of the CFC, supporting the assumption that the $\mathrm{D}$ detected deep in the material is due to co-deposition inside the CFC porous network. Raman microscopy indicates that the deposited layers correspond mainly to hard $a-C: D$ layers, data being nevertheless largely scattered, revealing a large variety from amorphous to disordered graphite-like carbon. Raman results are in agreement with the $D$ content of the layers measured with NRA (D/C $<30 \%$ ). D profiles obtained from SIMS and NRA have been compared and are consistent within their respective errors for the thin deposits but give inconsistent results in the case of the thick deposits and the erosion zone. The latter is attributed to the strong nonuniformity of the samples at the micrometer scale, as seen from the structural analysis, and to the probing sizes of the different methods (NRA integrates over typically a $1 \mathrm{~mm}$ diameter spot while SIMS is much more local). However, both methods give the same trend for gap measurements: the $D$ content decreases while going down the gap, but increases again at the gap bottom. Finally, SIMS was used to search for the DITS campaign markers $\left({ }^{13} \mathrm{C}\right.$ and $\left.{ }^{11} \mathrm{~B}\right)$, but the results are inconclusive so far, as the markers have only been found in 2 out of 7 measurements in the deposited layers.

\section{Acknowledgements}

We acknowledge the Euratom-CEA association, the Fédération de Recherche FR-FCM, the EFDA European Task Force on Plasma-Wall Interactions, and the French agency ANR for financial support. This work was carried out within the framework of the EFDA Task Force on Plasma-Wall Interactions. The views and opinions expressed herein do not necessarily reflect those of the European Commission.

\section{References}

[1] J. Roth et al., Plasma Phys. Contr. Fusion, 50, (2008) 103001

[2] B. Pegourié et al., J. Nucl. Mat., 390-391(2009) 550-555

[3] E. Tsitrone et al., Nucl. Fusion 49 (2009) 075011

[4] J.P. Coad, J. Likonen, M. Rubel et al., Nucl. Fus. 46 (2006) 350

[5] E. Delchambre et al., in: 30th EPS Conference on Controlled Fusion and Plasma Physics, ECA 27A, P-3.169, 2003.

[6] R. Dejarnac, J. Gunn, J. Nucl. Mater. 363-365, 560 (2007)

[7] N. Bernier et al., J. Nucl. Mater, 385, (2009) 601 
[8] H. Khodja et al., Nucl. Instr. Meth. B, 266, (2008),1425

[9] M. Mayer et al., Nucl. Instr. Meth. B, 267, (2009), 506

[10] C. Casiraghi, F. Piazza, A.C. Ferrari, D. Grambole, J. Robertson, Diamond and Relat. Mater. 14 (2005) 1098-1102

[11] A.C. Ferrari and J. Robertson, Phys. Rev. B 64 (2001) 075414

[12] N.M.J. Conway et al., Diamond and Relat. Mater. 9 (2000) 765-770

[13] M. Mayer: "SIMNRA User's Guide 6.04", Max-Planck-Institut für Plasmaphysik, Garching, Germany, 2008

[14] T. Schwarz-Selinger et al., Journal of Applied Physics, 86 (1999) 3988

[15] A. Litnovsky et al., J. Nucl. Mater, (2009) doi:10.1016/j.jnucmat.2009.01.097

[16] K. Krieger et al., J. Nucl. Mater, 363, (2007) 870

[17] J.T. Hogan et al., Contributions To Plasma Physics, 46 (2006) 750 


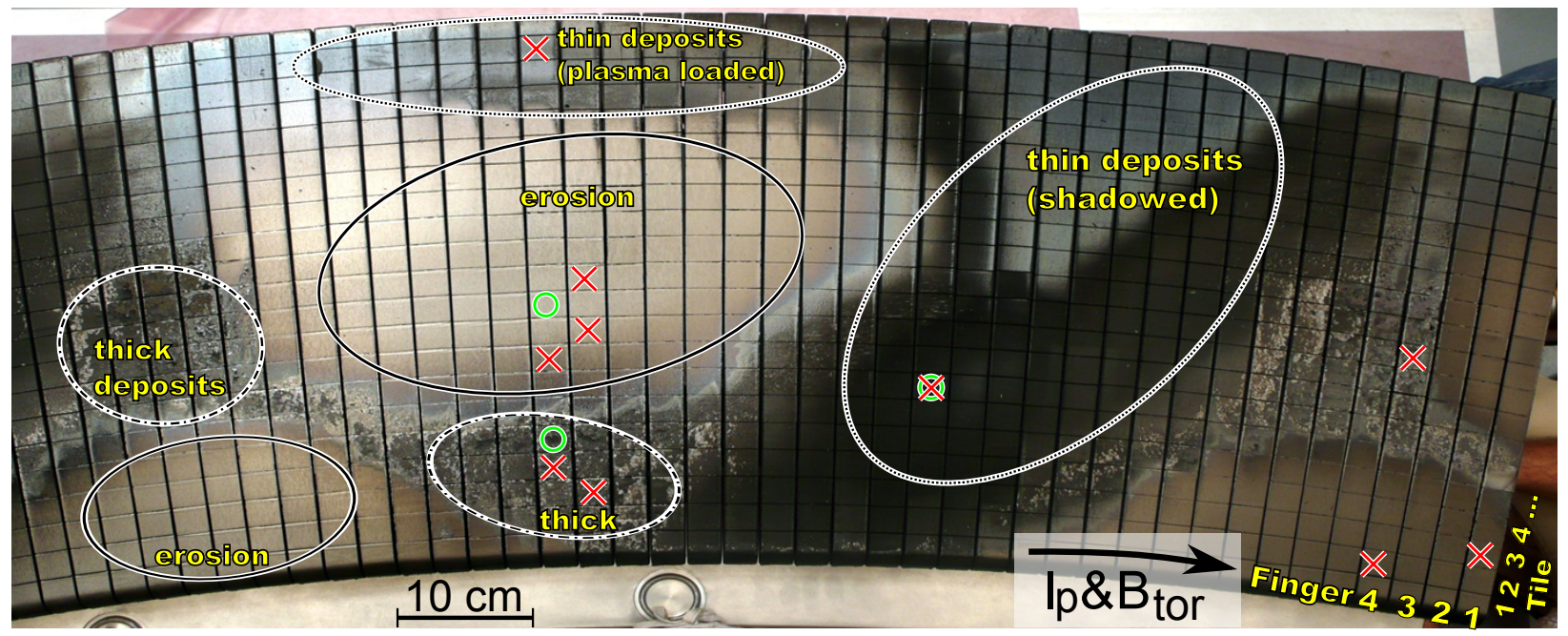

Figure 1: Erosion-deposition pattern of the extracted Tore Supra limiter sector. The different zones of interest (erosion, thin deposits, and thick deposits) are schematically indicated. Tiles selected for analyses are marked with red crosses for TDS, NRA or SIMS analyses, and with green circles for SEM and Raman analyses.
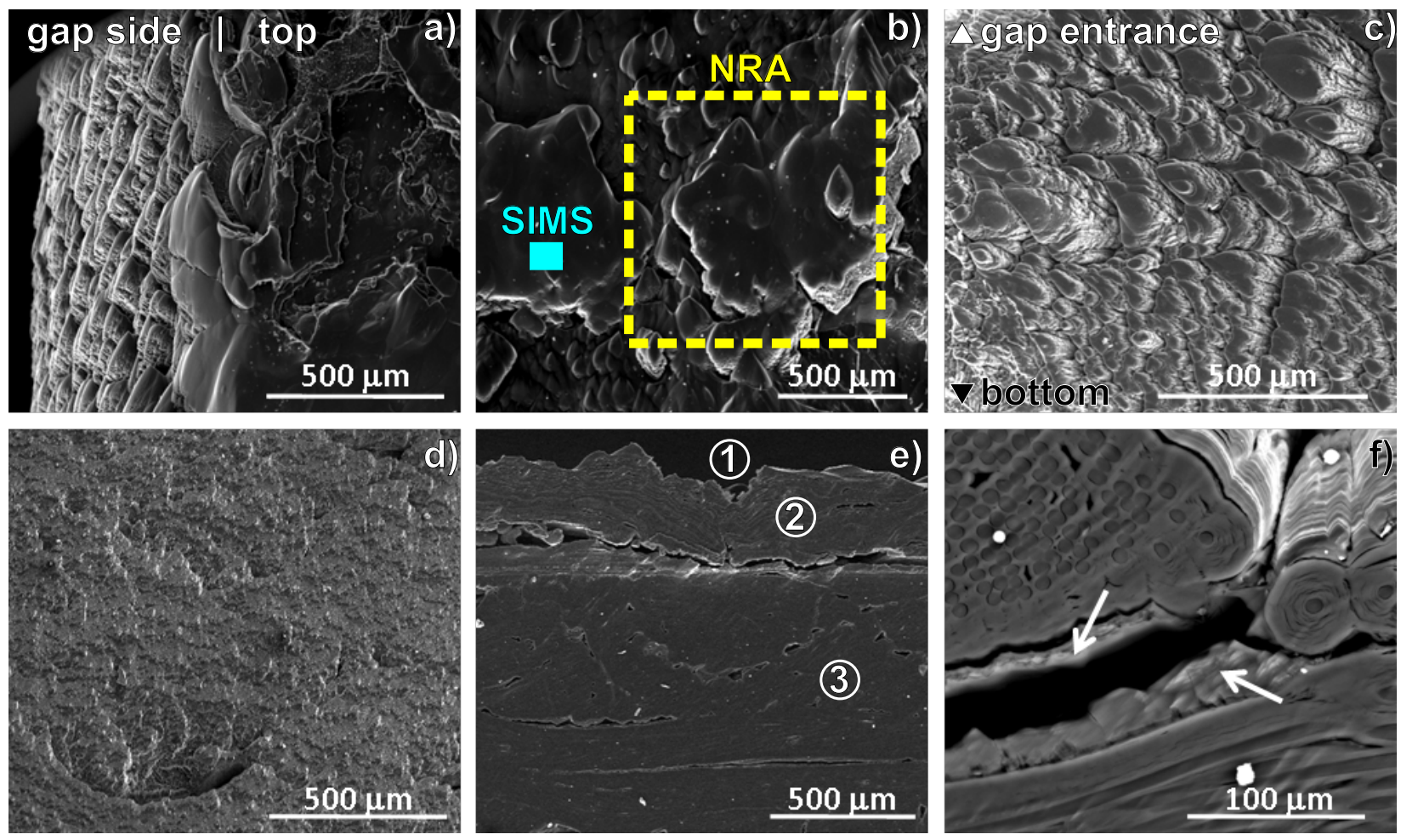

Figure 2: SEM images of the tiles surfaces. (a-c) Thick deposit tile (F27-T5): (a) top of the tile (right) and side corresponding to a poloidal gap (left) (b) another place of the top of the tile, with typical probing sizes for NRA and SIMS measurements, (c) front view of a poloidal gap, (d) top of the thin deposit tile (F17-T7) (e-f) top of the eroded tile (F27-T10): (e) top of the tile (3) showing the thick deposited layer loosely attached on a toroidal gap (2) and vacuum (1) (f) closeup view; arrows indicate deposits inside porosities. 


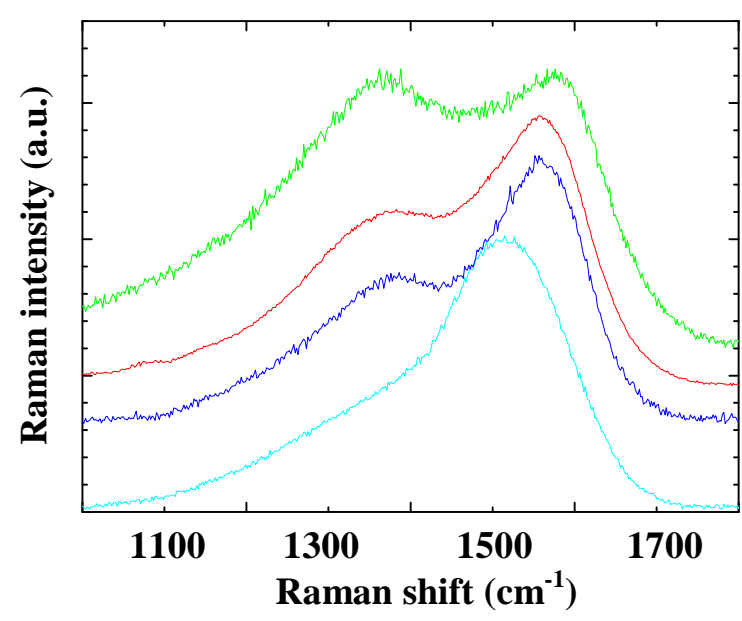

(a)

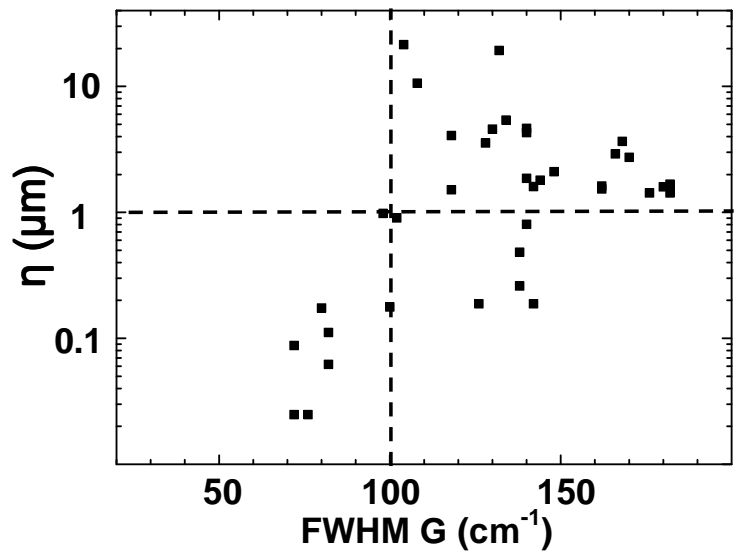

(b)

Figure 3: Raman spectroscopy: (a) typical spectra obtained for the limiter tiles (linear background already subtracted), (b) $\eta$ plotted as a function of the FWHM of the G band for the thick deposit tile, $\eta$ is a parameter which characterizes the D-content (see text). Dashed lines are guides for the eyes to indicate limit values for the $\eta$ parameter and for the $\mathrm{G}$ band width.

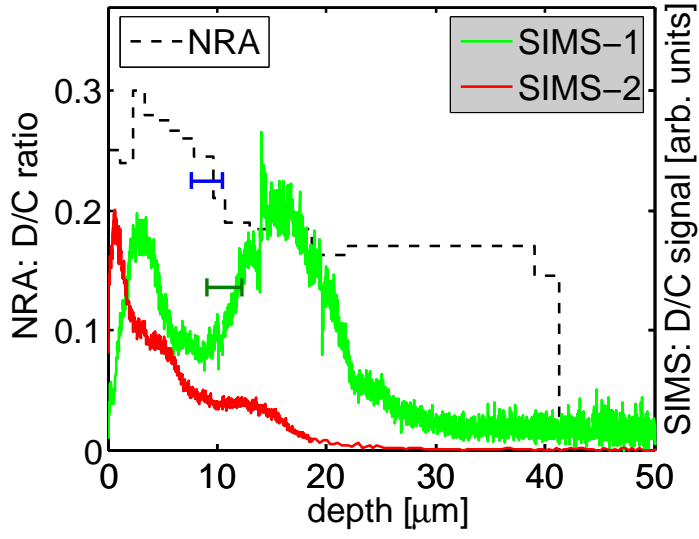

(a)

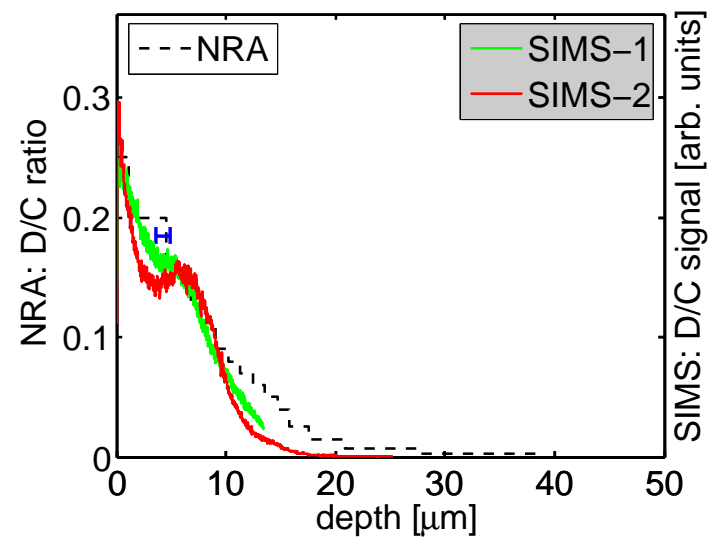

(b)

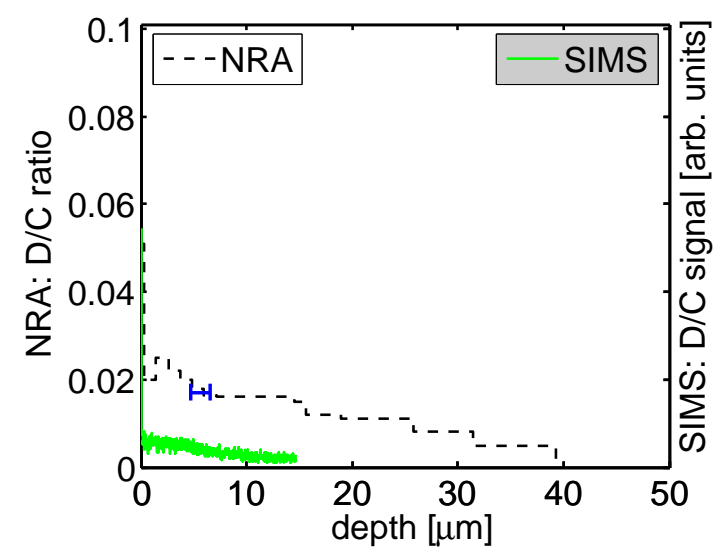

(c)

Figure 4: Deuterium depth profiles measured with SIMS and NRA on the top surface of the tiles: (a) thick deposit (tile F27-T4), (b) thin deposit (tile F17-T7) and (c) erosion zone (tile F27-T8). 


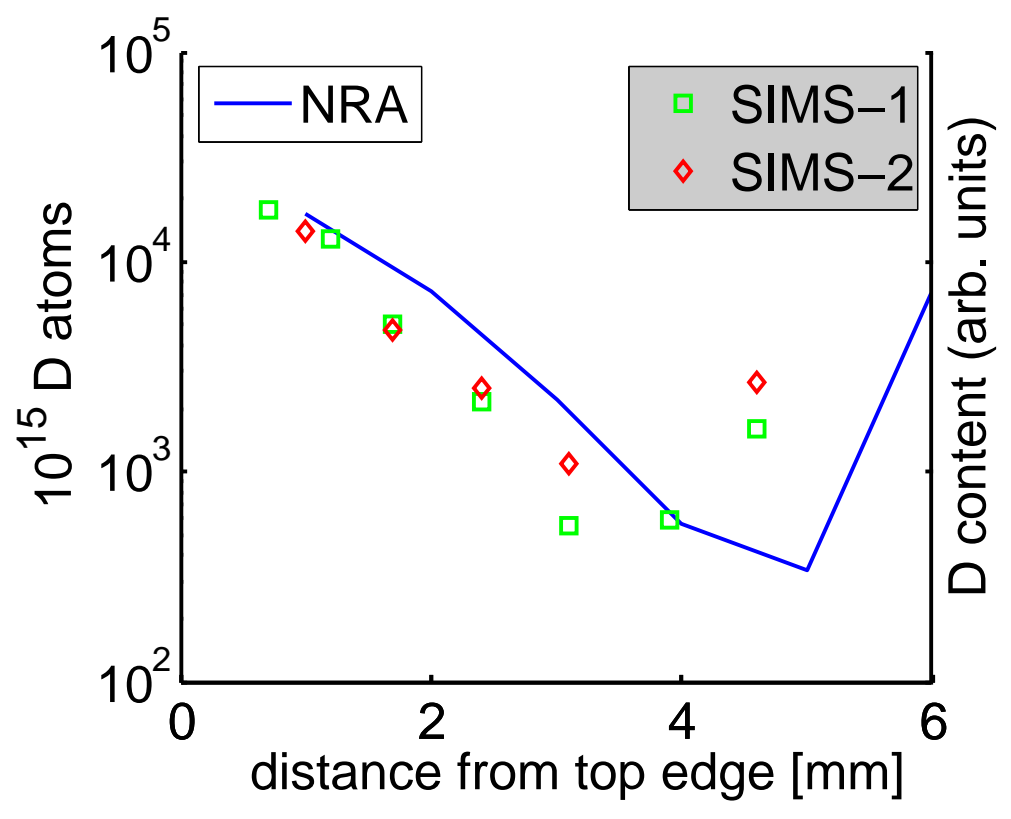

Figure 5: Deuterium distribution in the toroidal gap surface of tile F4-T2: comparison between SIMS and NRA integrated deuterium content as a function of the distance from the plasma facing top of the gap. 